Article

\title{
From G-Completeness to M-Completeness
}

\author{
Rachid Mecheraoui ${ }^{1, *} \mathbb{D}$, Aiman Mukheimer ${ }^{2} \mathbb{D}$ and Stojan Radenović ${ }^{3}$ \\ 1 Department of Mathematics, Faculty of Sciences and technology, Abbes Laghror University, \\ Khenchela 40000, Algeria \\ 2 Department of Mathematics and General Sciences, Prince Sultan University, Riyadh 11586, Saudi Arabia \\ 3 Department of Mathematics, College of Science, King Saud University, Riyadh 11451, Saudi Arabia \\ * Correspondence: rachid.Mecheraoui@yahoo.fr
}

Received: 27 May 2019; Accepted: 24 June 2019; Published: 27 June 2019

check for updates

\begin{abstract}
The purpose of this paper is to obtain a sufficient condition for a G-Cauchy sequence to be an $\mathrm{M}$-Cauchy sequence in fuzzy metric spaces. Our main result provides a partial answer to the open question posed by V. Gregori and A. Sapena. For application, we give a new fuzzy version of the Banach fixed point theorem.
\end{abstract}

Keywords: fuzzy metric space; G-Complete; M-Complete; Banach contraction; fixed point

\section{Introduction and Preliminaries}

Since 1965, or since Zadeh introduced the theory of fuzzy sets, many authors have been interested by the concept of fuzzy metric spaces. Kramosil and Michalek [1] introduced the notion of fuzzy metric space by generalizing the concept of the probabilistic metric space to the fuzzy situation. In 1994, A. George and P. Veeramani [2] modified the concept of fuzzy metric space introduced by Kramosil and Michalek and defined a Hausdorff topology on this fuzzy metric space. They modified the definition of Cauchy sequence in [3] (known in the literature as G-Cauchy sequence) to that known in the literature as M-Cauchy sequence (which is actually the natural definition of a Cauchy sequence). Song [4] insists that the definition of Cauchy sequence should be modified as: $\left\{x_{n}\right\}_{n}$, and is a Cauchy sequence if and only if $M\left(x_{n}, x_{n+p}, t\right) \rightarrow 1$, for all $t>0$, as $n \rightarrow \infty$ uniformly on $p \in \mathbb{N}$. He also adds that otherwise the definition of Cauchy sequence is incorrect. For more details, we refer the reader, for instance, to [5]. In 2002, V. Gregori and A. Sapena [6] obtained the following fuzzy versions of the Banach contraction principle:

Theorem 1 (Fuzzy Banach contraction theorem [6]). Let $(X, M, *)$ be an M-complete fuzzy metric space in which fuzzy contractive sequences are Cauchy. Let $T: X \longrightarrow X$ be a fuzzy contractive mapping. Then, $T$ has a unique fixed point.

Theorem 2 (Fuzzy Banach contraction theorem [6]). Let $(X, M, *)$ be a G-complete fuzzy metric space. Let $T: X \longrightarrow X$ be a fuzzy contractive mapping. Then, T has a unique fixed point.

A fuzzy contractive mapping and a fuzzy contractive sequence are defined as follows:

Definition 1. Let $(X, M, *)$ be a fuzzy metric space.

- A map $T: X \longrightarrow X$ is fuzzy contractive if there exists $k \in(0,1)$ such that

$$
\frac{1}{M(T x, T y, t)}-1 \leq k\left(\frac{1}{M(x, y, t)}-1\right)
$$


for all $x, y$ in $X, t>0$.

- A sequence $\left\{x_{n}\right\}_{n}$ in $X$ is fuzzy contractive if there exists $\left.k \in\right] 0,1[$ such that

$$
\frac{1}{M\left(x_{n+1}, x_{n+2}, t\right)}-1 \leq k\left(\frac{1}{M\left(x_{n}, x_{n+1}, t\right)}-1\right),
$$

for all $t>0, n$ in $\mathbb{N}$.

After that, the fuzzy-type Banach contraction principle has been generalized in many different directions over the years, see for instance [7-14].

As an open question, authors in [6] posed:

Question 1. Is every fuzzy contractive sequence a Cauchy sequence in the sense of George and Veeramani (or, simply, M-Cauchy)?

In [15], authors showed that in the case of fuzzy Menger spaces (see [1]) the answer is negative.

A partial answer of the previous question is given in this paper (Corollary 1). Furthermore, our main results (Theorems 3 and 4) provide a characterization of the G-Cauchy sequences which are M-Cauchy in fuzzy metric spaces. It should be emphasized that this characterization allows researchers to obtain many new results concerning the fixed points in fuzzy metric spaces, for application we give a new fuzzy version of the Banach contraction theorem (Theorem 5).

Before stating the main results, we need the following definitions.

Definition 2 (Schweizer and Sklar [16]). A binary operation $*:[0,1] \times[0,1] \longrightarrow[0,1]$ is called a continuous t-norm if it satisfies the following assertions:

(T1) * is commutative and associative;

(T2) $*$ is continuous;

(T3) $a * 1=$ a for all $a \in[0,1]$;

(T4) $a * b \leq c * d$ when $a \leq c$ and $b \leq d$, with $a, b, c, d \in[0,1]$.

Definition 3 (George and Veeramani [2]). A fuzzy metric space is an ordered triple $(X, M, *)$, such that $X$ denotes a nonempty set, * refers to a continuous $t$-norm, and $M$ is a fuzzy set on $X \times X \times(0, \infty) \longrightarrow(0,+\infty)$, satisfying the following conditions for all $x, y, z \in X$ and $t, s>0$ :

(F1) $M(x, y, t)>0$;

(F2) $M(x, y, t)=1$ if and only if $x=y$;

(F3) $M(x, y, t)=M(y, x, t)$;

(F4) $M(x, y, t) * M(y, z, s) \leq M(x, z, t+s)$;

(F5) $M(x, y,):.(0,+\infty) \longrightarrow(0,1]$ is left continuous.

Definition 4 (George and Veeramani [2]). Let $(X, M, *)$ be a fuzzy metric space. Then:

(i) A sequence $\left\{x_{n}\right\}_{n}$ converges to $x \in X$ if and only if $M\left(x_{n}, x, t\right) \longrightarrow 1$ as $n \longrightarrow+\infty$ for all $t>0$;

(ii) A sequence $\left\{x_{n}\right\}_{n}$ in $X$ is an $M$-Cauchy sequence if and only if for all $\varepsilon \in(0,1)$ and $t>0$, there exists $n_{0}$ such that $M\left(x_{n}, x_{m}, t\right)>1-\varepsilon$ for all $m, n \geq n_{0}$;

(iii) A sequence $\left\{x_{n}\right\}_{n}$ in $X$ is an $G$-Cauchy sequence if and only if for all $p \in \mathbb{N}, \varepsilon \in(0,1)$ and $t>0$, there exists $n_{0}$ such that $M\left(x_{n}, x_{n+p}, t\right)>1-\varepsilon$ for all $n \geq n_{0}$;

(iv) The fuzzy metric space is G-complete (M-complete resp. ) if every G-Cauchy (M-Cauchy resp.) sequence converges to some $x \in X$.

Remark 1. Every M-Cauchy sequence is a G-Cauchy sequence, but the converse is not true. Therefore, every G-complete fuzzy metric space is M-complete. 
Definition 5. Let $(X, M, *)$ be a fuzzy metric space.

1. We define $\Psi$ as the set of all functions $\varphi:(0,1) \times(0,1) \longrightarrow \mathbb{R}_{+}$satisfying

(A) $\varphi(t, t)<1$, for all $t \in(0,1)$;

(B) $\varphi$ is a continuous function and satisfies $\varphi(t, s) \geq 1$ implies that $t \geq s$, for all $t, s \in(0,1)$;

2. Let $\varphi \in \Psi$, a sequence $\left\{x_{n}\right\}_{n} \subset X$ is said to be a $\varphi$-fuzzy contractive sequence if

(C) $\varphi\left(M\left(x_{n}, x_{m}, t\right), M\left(x_{n+1}, x_{m+1}, t\right)\right) \geq 1$, for all $n, m \in \mathbb{N}$ and all $t>0$.

Example 1. Let $(X, M, *)$ be a fuzzy metric space. Let $T: X \longrightarrow X$ be self mapping such that

$$
\frac{1}{M(T x, T y, t)}-1 \leq k\left(\frac{1}{M(x, y, t)}-1\right),
$$

for all $x, y$ in $X, t>0$. Let $\varphi$ be a function defined by

$$
\varphi(t, s)=\frac{\frac{1}{s}-1}{\frac{1}{t}-1}, k \in(0,1) .
$$

Then, $\varphi \in \Psi$ and $\left\{T^{n} x\right\}$ are a $\varphi$-fuzzy contractive sequence for all $x \in X$.

Example 2. Let $(X, M, *)$ be a fuzzy metric space. Let $T: X \longrightarrow X$ be self mapping such that

$$
1-M(T x, T y, t) \leq k(1-M(x, y, t)),
$$

for all $x, y$ in $X, t>0$. Let $\varphi$ be a function defined by

$$
\varphi(t, s)=k \frac{1-s}{1-t}, k \in(0,1) .
$$

Then, $\varphi \in \Psi$ and $\left\{T^{n} x\right\}$ are a $\varphi$-fuzzy contractive sequence for all $x \in X$.

We use the following important technical lemma in the sequel.

Lemma 1. Let $(X, M, *)$ be a fuzzy metric space, $\left\{t_{n}\right\}_{n}$ is a decreasing sequence with positive terms such that $\lim _{n \rightarrow \infty} t_{n}=0$, and $\left\{x_{n}\right\}_{n}$ is a sequence in X. If $\left\{M\left(x_{n}, x_{n+1}, t\right)\right\}_{n}$ converges uniformly to 1, i.e.,

$$
\lim _{n \rightarrow \infty} \inf _{t>0} M\left(x_{n}, x_{n+1}, t\right)=1 \text {, }
$$

then, for all $x, y \in X$, we have

${ }^{*}\left\{M\left(x, y, t_{n}\right)\right\}_{n}$ is a decreasing and convergent sequence.

* Putting

$$
\tilde{M}(x, y, t)= \begin{cases}M(x, y, t) & \text { if } t \in(0, \infty) \\ \lim _{n \rightarrow \infty} M\left(x, y, t_{n}\right) & \text { if } t=0\end{cases}
$$

we get

$$
\lim _{n \rightarrow \infty} \tilde{M}\left(x_{n}, x_{n+1}, 0\right)=1 .
$$

Proof. Let $x, y \in X$ and $\left\{t_{n}\right\}_{n} \subset \mathbb{R}_{+}^{*}$ be a decreasing sequence such that $\lim _{n} t_{n}=0$. Since $t \mapsto M(x, y, t)$ is a bounded non-decreasing function [3], we deduce that $\left\{M\left(x, y, t_{n}\right)\right\}_{n}$ is a decreasing and convergent sequence. Let $\left\{x_{n}\right\}_{n} \subset X$ be a sequence with

$$
\lim _{n \rightarrow \infty} \inf _{t>0} M\left(x_{n}, x_{n+1}, t\right)=1,
$$


This means that for every $\varepsilon>0$ there exists $n_{0} \in \mathbb{N}$, such that for all $n \geq n_{0}$ and for all $t>0$ we have $M\left(x_{n}, x_{n+1}, t\right)>1-\frac{\varepsilon}{2}$. But

$$
1-\tilde{M}\left(x_{n}, x_{n+1}, 0\right)=\lim _{k \rightarrow \infty}\left(1-M\left(x_{n}, x_{n+1}, t_{k}\right)\right)
$$

For $n \geq n_{0}$, using the above inequality, we obtain $1-\tilde{M}\left(x_{n}, x_{n+1}, 0\right) \leq \frac{\varepsilon}{2}<\varepsilon$, hence $\tilde{M}\left(x_{n}, x_{n+1}, 0\right)>1-\varepsilon$, which proves the claim.

Remark 2. Condition (3) is essential and can not be replaced by

$$
\lim _{n \rightarrow \infty} M\left(x_{n}, x_{n+1}, t\right)=1, \forall t>0 .
$$

The examples below shows that clearly.

Example 3. Let $(X, d)$ be a metric space. Let $M$ be the fuzzy set defined on $X \times X \times[0,+\infty]$ by

$$
M(x, y, t)=\frac{t}{t+d(x, y)} .
$$

Let $t_{n}=\frac{1}{n}$ for all $n \in \mathbb{N}^{*}$, we obtain $\lim _{n} M\left(x, y, t_{n}\right)=\theta^{x, y}$, where

$$
\theta^{x, y}=\left\{\begin{array}{lll}
1 & \text { if } & x=y \\
0 & \text { if } & x \neq y
\end{array}\right.
$$

On the other hand, let $\left\{x_{n}\right\}_{n}$ be a convergent sequence in $X$ such that $x_{n} \neq x_{n+1}$ for all $n$ large enough. For all $t>0$, we get $\lim _{n} M\left(x_{n}, x_{n+1}, t\right)=1$ and $\lim _{n} \inf _{t>0} M\left(x_{n}, x_{n+1}, t\right)=0$, while $\lim _{n} M\left(x_{n}, x_{n+1}, 0\right)=0$.

Example 4. On $X=[1,+\infty]$, we define for all $x, y \in X, t>0$ the fuzzy set defined on $X \times X \times[0,+\infty]$ by

$$
M(x, y, t)=\frac{\min \{x, y\}+t}{\max \{x, y\}+t} .
$$

Here, for all convergent sequence $\left\{x_{n}\right\}_{n}$ in $X$ and all $t>0$ we have

$$
\begin{aligned}
\liminf _{n} M\left(x_{n}, x_{n+1}, t\right) & =\liminf _{n} \frac{\min \left\{x_{n}, x_{n+1}\right\}+t}{\max \left\{x_{n}, x_{n+1}\right\}+t} \\
& =\lim _{n} \frac{\min \left\{x_{n}, x_{n+1}\right\}}{\max \left\{x_{n}, x_{n+1}\right\}} \\
& =1,
\end{aligned}
$$

and for all sequence $\left\{t_{n}\right\}_{n}$ in $\mathbb{R}_{+}$verifying $\lim _{n} t_{n}=0$ and all $x, y \in X$ we have $\lim _{n} M\left(x, y, t_{n}\right)=\tilde{M}(x, y, 0)$ and $\lim _{n} \tilde{M}\left(x_{n}, x_{n+1}, 0\right)=1$.

\section{Results}

Now, we state our main result:

Theorem 3. Let $(X, M, *)$ be a fuzzy metric space. Let $\varphi \in \Psi$ and $\left\{x_{n}\right\}_{n} \subset X$ be a $\varphi$-fuzzy contractive sequence, then

(I)- $\lim _{n \rightarrow \infty} M\left(x_{n}, x_{n+1}, t\right)=1$, for all $t>0$;

(II)- the sequence $\left\{x_{n}\right\}_{n}$ is a G-Cauchy sequence;

(III)- if $\lim _{n \rightarrow \infty} M\left(x_{n}, x_{n+1}, t\right)=1$ uniformly on $(0,+\infty)$, then the sequence $\left\{x_{n}\right\}_{n}$ is an M-Cauchy sequence. 
Proof. The proof is divided into two cases. Case 1: If there exists $n_{0} \in \mathbb{N}$ such that $x_{n_{0}}=x_{n_{0}+1}$, then it follows that $x_{n}=x_{n_{0}}$ for all $n>n_{0}$ and the three parts in theorem 2.1 are, clearly, fulfilled. Case 2: We assume that the relationship $x_{n} \neq x_{n+1}$ holds true for each $n$. Let $t>0$ and $n \in \mathbb{N}$, by assumption (C) in definition (1.9) we obtain

$$
\varphi\left(M\left(x_{n+1}, x_{n+2}, t\right), M\left(x_{n}, x_{n+1}, t\right)\right) \geq 1,
$$

and by assumption (B) we deduce that

$$
M\left(x_{n+1}, x_{n+2}, t\right) \geq M\left(x_{n}, x_{n+1}, t\right) .
$$

Consequently, the sequence $\left\{M\left(x_{n}, x_{n+1}, t\right)\right\}_{n}$ is non-decreasing and bounded, for all $t>0$, so, it converges to some point $a_{t}$ in $[0,1]$. We claim that

$$
a_{t}=\lim _{n} M\left(x_{n}, x_{n+1}, t\right)=1,
$$

for all $t>0$. Indeed, $\left\{M\left(x_{n}, x_{n+1}, t\right)\right\}_{n}$ is non-decreasing so, obviously, $a_{t} \neq 0$. On the other hand, using the continuity of the function $\varphi$ (assumption (B)) and relation (6) it follows that $\varphi\left(a_{t}, a_{t}\right) \geq 1$. But, by assumption (A), if $a_{t} \in(0,1)$, we get $\varphi\left(a_{t}, a_{t}\right)<a_{t}(\leq 1)$. So, we deduce that $a_{t}=1$, for all $t>0$. Let $p \in \mathbb{N}$, by assumption (F4) we obtain

$$
M\left(x_{n}, x_{n+p}, t\right) \geq M\left(x_{n}, x_{n+1}, \frac{t}{p}\right) * M\left(x_{n+1}, x_{n+2}, \frac{t}{p}\right) * \cdots * M\left(x_{n+p-1}, x_{n+p}, \frac{t}{p}\right) .
$$

Using relation (8) and assumption (T2) we get

$$
\lim _{n} M\left(x_{n}, x_{n+p}, t\right)=1,
$$

for all $p \in \mathbb{N}$-which means that $\left\{x_{n}\right\}_{n}$ is a G-Cauchy sequence. Now, we suppose that $\lim _{n \rightarrow \infty} M\left(x_{n}, x_{n+1}, t\right)=1$ uniformly on $(0,+\infty)$. To prove that $\left\{x_{n}\right\}_{n}$ is an M-Cauchy sequence, we assume to the contrary. Then, there exists some $\varepsilon \in(0,1)$ and some $t_{0}>0$ such that for every $p \in \mathbb{N}$ we find $m_{p} \geq n_{p} \geq p$ such that $M\left(x_{m_{p}}, x_{n_{p}}, t_{0}\right) \leq 1-\varepsilon$. Clearly, here $m_{p}>n_{p}$. We choose minimal $m_{p}$ and $n_{p}$ satisfying the above conditions, it yields

$$
M\left(x_{m_{p}}, x_{n_{p}}, t_{0}\right) \leq 1-\varepsilon \text { and } M\left(x_{m_{p}-1}, x_{n_{p}}, t_{0}\right)>1-\varepsilon .
$$

Taking into account the left-continuity of the function $t \mapsto M(x, y, t)$ and the fact that $M\left(x_{m_{p}-1}, x_{n_{p}}, t_{0}\right)>$ $1-\varepsilon$, we can choose $q_{0} \in \mathbb{N}$ such that

$$
M\left(x_{m_{p}-1}, x_{n_{p}}, t_{0}-\frac{1}{q_{0}}\right)>1-\varepsilon
$$

Let $\tilde{M}: X \times X \times[0, \infty) \longrightarrow[0,1]$ be defined as in lemma 1 (with $t_{n}=\frac{1}{n+1}$ for all $n \in \mathbb{N}$ ). By virtue of assumption (F4) and relation (9) it follows that

$$
\begin{aligned}
1-\varepsilon & \geq M\left(x_{m_{p}}, x_{n_{p}}, t_{0}\right) \\
& \geq M\left(x_{m_{p}}, x_{m_{p}-1}, \frac{1}{q_{0}}\right) * M\left(x_{m_{p}-1}, x_{n_{p}}, t_{0}-\frac{1}{q_{0}}\right),
\end{aligned}
$$


and by relations (10), limit (5) and the fact that $M\left(x, y, t_{n}\right) \downarrow_{n \rightarrow \infty} \tilde{M}(x, y, 0)$ (Lemma 1), yields

$$
\begin{aligned}
1-\varepsilon \geq & M\left(x_{m_{p}}, x_{n_{p}}, t_{0}\right) \\
\geq & \tilde{M}\left(x_{m_{p}}, x_{m_{p}-1}, 0\right) *(1-\varepsilon) \\
& \longrightarrow p \longrightarrow \infty 1-\varepsilon .
\end{aligned}
$$

Consequently

$$
\lim _{p \longrightarrow \infty} M\left(x_{m_{p}}, x_{n_{p}}, t_{0}\right)=1-\varepsilon .
$$

Suppose that for all $p_{1} \geq 0$, there exists $p \geq p_{1}$ such that $M\left(x_{m_{p}+1}, x_{n_{p}+1}, t_{0}\right) \leq 1-\varepsilon$ means-having in mind relations (7),(11)—that the sequence $\left\{x_{n}\right\}_{n}$ has two sub-sequences $\left\{x_{n_{p}}\right\}_{p}$ and $\left\{x_{m_{p}}\right\}_{p}$, verifying

$$
\lim _{p \longrightarrow \infty} M\left(x_{m_{p}}, x_{n_{p}}, t_{0}\right)=\lim _{p \longrightarrow \infty} M\left(x_{m_{p}+1}, x_{n_{p}+1}, t_{0}\right)=1-\varepsilon,
$$

(for simplicity, we have saved the same notation for the sub-sequences). Now, we suppose that there exists $p_{1} \geq 0$ such that $M\left(x_{m_{p}+1}, x_{n_{p}+1}, t_{0}\right)>1-\varepsilon$ for all $p \geq p_{1}$. Suppose that $\lim _{p} M\left(x_{m_{p}+1}, x_{n_{p}+1}, t_{0}\right) \neq$ $1-\varepsilon$, i.e., there exists $\alpha>0$-for all $n_{0} \in \mathbb{N}$, there exists $p>n_{0}$ such that

$$
M\left(x_{m_{p}+1}, x_{n_{p}+1}, t_{0}\right)>\alpha+(1-\varepsilon) .
$$

With $q \in \mathbb{N}$ satisfying $M\left(x_{m_{p}+1}, x_{n_{p}+1}, t_{0}-\frac{1}{q}\right)>\alpha+(1-\varepsilon)$, we obtain

$$
\begin{aligned}
1-\varepsilon \geq & M\left(x_{m_{p}}, x_{n_{p}}, t_{0}\right) \\
\geq & M\left(x_{m_{p}}, x_{m_{p}+1}, \frac{1}{2 q}\right) * M\left(x_{m_{p}+1}, x_{n_{p}+1}, t_{0}-\frac{1}{q}\right) \\
& * M\left(x_{n_{p}+1}, x_{n_{p}}, \frac{1}{2 q}\right) \\
\geq & \tilde{M}\left(x_{m_{p}}, x_{m_{p}+1}, 0\right) *[\alpha+(1-\varepsilon)] * \tilde{M}\left(x_{n_{p}+1}, x_{n_{p}}, 0\right) \\
& \longrightarrow p \rightarrow \infty \alpha+(1-\varepsilon),
\end{aligned}
$$

contradiction, then

$$
\lim _{p} M\left(x_{m_{p}+1}, x_{n_{p}+1}, t_{0}\right)=1-\varepsilon .
$$

On the other hand, the fact that $\left\{x_{n}\right\}_{n}$ is a $\varphi$-fuzzy contraction sequence means

$$
\varphi\left(M\left(x_{n_{p}+1}, x_{m_{p}+1}, t\right)\right), M\left(x_{n_{p}}, x_{m_{p}}, t\right) \geq 1 .
$$

for all $p \in \mathbb{N}$ and $t>0$. Using limits (11), (12), (13), and relation (14) with assumptions (A,B) we get a clear contradiction:

$$
1>\varphi(1-\varepsilon, 1-\varepsilon) \geq 1,
$$

which means that $\left\{x_{n}\right\}_{n}$ is an M-Cauchy sequence. This achieves the proof.

From this theorem, we can easily get the following results (we omit their proofs for simplicity):

Corollary 1. Every fuzzy contractive sequence verifying condition (3) is a Cauchy sequence in George and Veeramani's sense.

Proof. Derives directly from Theorem 3.

Theorem 4. Let $(X, M, *)$ be a fuzzy metric space and $\left\{x_{n}\right\}_{n}$ be a G-Cauchy sequence in $X$. If 
(I)- $\lim _{n \rightarrow \infty} M\left(x_{n}, x_{n+1}, t\right)=1$ uniformly on $(0,+\infty)$;

(II)- there exists $\varphi$ in $\Psi$ such that $\left\{x_{n}\right\}_{n}$ is a $\varphi$-fuzzy contractive.

Then $\left\{x_{n}\right\}_{n}$ is an M-Cauchy sequence.

Proof. Derives directly from Theorem 3.

Theorem 5 (Fuzzy Banach contraction theorem). Let $(X, M, *)$ be an $M$-complete fuzzy metric space. Let $T: X \longrightarrow X$ be a fuzzy contractive mapping and verifying condition (3). Then, $T$ has a unique fixed point.

Proof. Derives directly from Theorem 3.

\section{Conclusions}

In this paper, we introduced the notion of $\varphi$-fuzzy contractive sequence and discussed the relation between a G-Cauchy sequence and an M-Cauchy sequence. We established that, under some conditions, any G-Cauchy sequence is an M-Cauchy sequence. For application, we gave a new fuzzy version of the Banach Contraction Principal. This brings us a natural question: How large is the class of maps that generate $\varphi$-fuzzy contractive sequences (in the sense of: $\left\{T^{n} x\right\}_{n}$ is a $\varphi$-fuzzy contractive sequence) in comparison with the existing generalizations of contraction mappings?

Author Contributions: Conceptualization, R.M.; methodology, R.M.; software, R.M.; validation, R.M., A.M. and S.R.; formal analysis, R.M.; investigation, R.M.; resources, R.M.; data curation, R.M.; writing-original draft preparation, R.M., A.M. and S.R.; writing-review and editing, R.M., A.M. and S.R.; visualization, R.M., A.M. and S.R.; supervision, R.M., A.M. and S.R.; project administration, R.M., A.M. and S.R.

Funding: This research received no external funding.

Acknowledgments: The second author would like to thank Prince Sultan University for funding this work through the Nonlinear Analysis Methods in Applied Mathematics (NAMAM) research group, group number RG-DES-2017-01-17.

Conflicts of Interest: The authors declare no conflict of interest.

\section{References}

1. Kramosil, I.; Michalek, J. Fuzzy metrics and statistical metric spaces. Kybernetika 1975, 11, 336-344.

2. George, A.; Veeramani, P. On some results in fuzzy metric spaces. Fuzzy Sets Syst. 1994, 64, $395-399$. [CrossRef]

3. Grabiec, M. Fixed points in fuzzy metric spaces. Fuzzy Sets Syst. 1988, 27, 385-389. [CrossRef]

4. Song, G. Comments on A common fixed point theorem in a fuzzy metric space. Fuzzy Sets Syst. 2003, 135, 409-413. [CrossRef]

5. Vasuki, R.; Veeramani, P. Fixed point theorems and Cauchy sequences in fuzzy metric spaces. Fuzzy Sets Syst. 2003, 135, 415-417. [CrossRef]

6. Gregori, V.; Sapena, A. On fixed-point theorems in fuzzy metric spaces. Fuzzy Sets Syst. 2002, 125, $245-252$. [CrossRef]

7. Habibi, F.; Birgani, O.; Koppelaar, H.; Radenović, S. Using fuzzy logic to improve the project time and cost estimation based on Project Evaluation and Review Technique (PERT). J. Proj. Manag. 2018, 3, 183-196. [CrossRef]

8. Azam, A.; Khan, M.N.A.; Mehmood, N.; Radenović, S.; Došenović, T. Coincidence point of L-fuzzy sets endowed with graph. Rev. Real Acad. Cienc. Exactas Fisicasy Nat. Ser. A Matemáticas 2018, 112, 915-931. [CrossRef]

9. Sedghi, S.; Shobkolaei, N.; Došenović, T.; Radenović, S. Suzuki-type of common fixed point theorems in fuzzy metric spaces. Math. Slovaca 2018, 68, 451-462. [CrossRef]

10. Azam, A.; Mehmood, N.; Rashid, M.; Radenović, S. Fuzzy fixed point theorems in ordered cone metric spaces. Filomat 2015, 29, 887-896. [CrossRef]

11. Chauhan, S.; Shatanawi, W.; Kumar, S.; Radenović, S. Existence and uniqueness of fixed points in modified intuitionistic fuzzy metric spaces. J. Nonlinear Sci. Appl. 2014, 7, 28-41. [CrossRef] 
12. Chauhan, S.; Radenović, S.; Imdad, M.; Vetro, C. Some integral type fixed point theorems in non-Archimedean Menger PM-spaces with common property (EA) and application of functional equations in dynamic programming. Rev. Real Acad. Cienc. Exactas Fisicasy Nat. Ser. A Matemáticas 2014, 108, 795-810. [CrossRef]

13. S Chauhan, S.; Bhatnagar, S.; Radenović, S. Common fixed point theorems for weakly compatible mappings in fuzzy metric spaces. J. Appl. Math. 2013, 68, 87-98.

14. Došenović, T.; Rakic, D.; Caric, B.; Radenović, S. Multivalued generalizations of fixed point results in fuzzy metric spaces. Nonlinear Anal. Model. Control. 2015, 21, 211-222.

15. Mihet, D. A Banach contraction theorem in fuzzy metric spaces. Fuzzy Sets Syst. 2004, 144, 431-439. [CrossRef]

16. Schweizer, B.; Sklar, A. Statistical metric spaces. Pac. J. Math. 1960, 10, 314-334. [CrossRef]

(C) 2019 by the authors. Licensee MDPI, Basel, Switzerland. This article is an open access article distributed under the terms and conditions of the Creative Commons Attribution (CC BY) license (http://creativecommons.org/licenses/by/4.0/). 